\title{
Transcription, Translation, and Immunolocalization of ODVP-6E/ODV-E56 and p74 Proteins: Two Highly Conserved ODV-associated Envelope Proteins of Choristoneura fumiferana Granulovirus
}

\author{
Kianoush Khajeh Rashidan*, Nasha Nassoury ${ }^{\dagger}$, Paresa N. Giannopoulos and Claude Guertin \\ Institut national de la recherche scientifique-Institut Armand-Frappier 531 Blvd des Prairies, H7V 1B7, Laval, Canada \\ 'Université de Montréal, 4101 Sherbrook east, H1X 2B2 Montreal, Canada
}

Received 16 June 2004, Accepted 19 August 2004

\begin{abstract}
Choristoneura fumiferana granulovirus (ChfuGV) infection results two types of enveloped virions: Occlusion-derived virus (ODV) and budded virus (BV). Structural proteins ODVP-6E/ODV-E56 and p74 are two major conserved ODV-associated proteins that may be involved in the initiation of viral infection cycle in susceptible host insect larvae. This study presents the characterization of ChfuGV $o d v p-6 e / o d v-e 56$ and p74 transcription and translation as well as immunolocalization of these proteins in the occluded ChfuGV virion. Our results revealed that the transcription of odvp-6e/odv-e56 and p74 genes, both, start at 24 hours post infection (h p.i.). Using monospecific polyclonal antibodies made against ODVP-6E/ODV-E56 and p74 we demonstrated that these proteins are both expressed late in infection ( $24 \mathrm{~h}$ p.i.). Immunogold labeling using antisera against ODVP-6E/ODV-E56 and p74 proteins demonstrated that ODVP-6E/ODV-E56 and p74 proteins are both associated with the ODV envelop of ChfuGV.
\end{abstract}

Keywords: Baculovirus, Choristoneura fumiferana granulovirus, ODV envelop associated proteins, ODVP-6E/ ODV-E56 and p74 proteins, Transcription, Translation and immunolocalization

\section{Introduction}

The Baculoviridae are complex and diverse family of entemopathogens that infect especially insects belonging largely to the orders Lepidoptera, Dieptera and Hymenoptera (Crook, 1991). This family of viruses contains a doublestranded, supercoiled DNA genome which is packed in a rod-

*To whom correspondence should be addressed.

Tel: 514-987-3000; Fax: 514-987-4647

E-mail: kiarashidan@juno.com shaped enveloped nucleocapsid (Miller, 2001). Members of Baculoviridae family are comprised of two genera: the nucleopolyhedroviruses (NPVs) and the granuloviruses $(\mathrm{GVs})$. Granuloviruses have been well characterized at the cytological level and their application for pest control in agriculture and forestry has been fairly successful (Crook, 1991; Moscardi, 1999). The spruce budworm, Choristoneura fumiferana, is the most devastating coniferous tree pest in eastern Canada and the United States. C. fumiferana granulovirus (ChfuGV) is being considered as an alternative biological insecticide due to their specificity for its insect host.

The infection cycle initiates after $C$. fumiferana (spruce budworm) larvae ingest the occluded virions. The proteinous matrix of occlusion bodies dissociates under the alkaline $\mathrm{pH}$ condition in the midgut and liberates enveloped virions known as occlusion-derived virus (ODV) (Miller, 2001). Throughout the baculoviral infection cycle, two virion phenotypes are produced: budded virus (BV) and occlusion-derived virus (ODV). These phenotypes can be distinguished by the origin of their envelopes: BVs obtain their envelopes during budding process through the plasma membrane of the infected cells (Blissard and Rohrmann, 1989; Adams and Mc Clintock, 1991; Blissard, 1996) and ODVs attain their envelope inside the nucleus of the infected cell from intracellular microvesicules within the invaginated inner nuclear membrane (Braunagel and Summers, 1994; Hong et al., 1994; Braunagel et al., 1996a). The BV phenotype is responsible for the spread of infection from cell to cell inside the infected insect host body (Tanada and Hess, 1991; Blissard, 1996). The ODV phenotype is produced in late steps of infection within the nucleus of infected cells and is subsequently occluded in a proteinous matrix to form the occluded virions. ODVs transmit infection exclusively from insect to insect, while the BVs spread infection from infected cells to healthy cells inside the infected host (Blissard, 1996).

The attachment of ODVs to microvilli of insect midgut cells followed by the virus penetration is considered as vital 
steps for the initiation of infection cycle in the host. The protein(s) that could participate in these steps have not yet been identified but there are evidences suggesting that the ODV penetration is a non-endocytotic process and requires interaction of the virion envelope proteins with midgut cell surface proteins which eventually leads to membrane fusion (Horton and Burand, 1993).

In ChfuGV, like other baculoviruses, the ODV phenotype has a complex structure. Baculoviral ODV envelopes contain a number of proteins such as: VP17 (Funk and Consigli, 1993), ODV-E25 (Russell and Rohrmann, 1993), ODV-E35 (Braunagel et al., 1996b), GP41 (Whitford and Faulkner, 1993), p74 (Kuzio et al., 1989; Rashidan et al., 2003), ODVE18 (Braunagel et al., 1996b), ODV-E66 (Hong et al., 1994), and ODVP-6E/ODV-E56 (Braunagel et al., 1996a; Theilmann et al., 1996; Rashidan et al., 2002). One or more of these proteins could participate in the adsorption, fusion and penetration process.

Baculoviral ODVP-6E/ODV-E56 protein is an extremely conserved protein (Rashidan et al., 2002); the elevated extent of primary and secondary structures preservation, even between two distantly related members of Baculoviridae family, points out the significance of ODVP-6E/ODV-E56 and suggest a probable major role for this protein during the infection cycle. The deduced amino acid composition of ChfuGV ODVP-6E/ODV-E56 indicates the presence of three potential $N$ - glycosylation $\mathrm{N}-\{\mathrm{P}\}-[\mathrm{ST}]-\{\mathrm{P}\}$ sites located at residues 59, 179, and 279 . Two hydrophobic membranespanning regions and six conserved cysteine residues between two hydrophobic membrane-spanning regions has been identified in ChfuGV ODVP-6E/ODV-E56 and those of other baculoviruses (Rashidan et al., 2002). These highly conserved cysteine residues have been speculated to be implicated in the creation of disulfide bridges that ultimately form the right folding of baculoviral ODVP-6E/ODV-E56 proteins (Rashidan et al., 2002).

Baculoviral p74 protein is another major protein associated with the ODV envelop. Studies on this protein in Autographa californica multicapsid nucleopolyhedrovirus (AcMNPV) (prototype of the Baculoviridae family) revealed that p74 protein is exposed on the virion surface with an N-terminal located outside of the ODV envelope and a transmembrane Cterminal that acts as an anchor (Faulkner et al., 1997; Slack et al., 2001). It has been suggested that the baculoviral p74 protein may play a crucial role in the virulence of the virus (Kuzio et al. 1989; Faulkner et al., 1997). It has been shown that although p74 null mutants fail to initiate infection in insect larvae when virus are fed orally, they are infectious once injected into the haemocoel of the larvae (Faulkner et al., 1997). These observations suggest $\mathrm{p} 74$ protein is essential for initiation of infection and its presence is an essential constituent for attachment and /or fusion of the ODV envelope to the membrane of midgut epithelial cells. $\mathrm{Wu}$ and his associates have demonstrated that baculovirus p74 gene is a species-specific gene (Wu et al., 2003). They have shown that the situation of AcMNPV p74 knock out with a p74 gene from Spodoptera litura nucleopolyhedrovirus (SINPV), would inhibit recombinant AcMNPV from infecting the Argyrogramma agnata larvae (Wu et al., 2003).

By nucleotide and amino acid sequence analyses it has been predicted that ChfuGV ODVP-6E/ODV-E56 and p74 proteins were ODV-envelope associated proteins that are expressed during the late stages of infection cycle (Rashidan et al., 2002; 2003). Here, we confirm, by transcriptional and translational analyses, the late expression of these proteins. We also examine the validity of our computer assisted predictions which classified ChfuGV ODVP-6E and p74 as ODV associated envelop proteins by the means of Electron microscopy immunolocalization.

\section{Materials and Methods}

RNA extraction and analysis of transcription Total RNA was extracted from the fat body of the infected fourth instar larvae at different hours post infection (h p.i.). Extraction was carried out using TRIZOL reagent (Stratagene, La Jolla, USA) according to the protocol provided by supplier. Extracted RNAs were quantified and stored under RNAguard and ethanol at $-70^{\circ} \mathrm{C}$. RNA samples (12 ug) were loaded on formaldehyde-agarose gels and electrophoresis was carried out. RNA was either visualized by ethidium bromide staining or transferred to Hybond N nylon membrane by capillary blotting as described in standard protocols (Sambrook et al., 1989). Transcripts of odvp-6e/odv-e56 and p74 genes were detected by Northern hybridization using ${ }^{32} \mathrm{P}$ [dATP] DNA probes labeled by random priming (Promega, Madison, USA).

The probe for $o d v p-6 e / o d v-e 56$ was generated using a 758bp PCR fragment as template. The fragment was amplified using the following two odvp-6elodv-e56 internal primers: FRe56-TTT ACG GGT CTA AGA CGC AC, and RVe56- ACG ACA AAC ACT TTG TTC. Meanwhile the probe for p74 was generated using a 1161bp PCR fragment as template, amplified using the following two p74 internal primers: FRp74: CCT ATT GTC GCC GCT TTG GTC GTT CTG and RVp74: GT GGT AGA CGC CAA ACT AGC ACC.

Temporal transcriptional activity of the odvp-6e/odv-e56 transcripts were examined by RT-PCR using total RNA isolated from ChfuGV-infected larvae collected at various times p.i. RTPCR reactions were realized with an one-step Reverse Transcriptase PCR (Invitrogen, Life technologies, San Diego, USA), as provided by manufacturer, using two internal specific primers for the odvp$6 e / o d v-e 56$ gene which have been mentioned before (FRe56- and $R V e 56$ ) . Using these primers a $758 \mathrm{bp}$ fragment of the target gene was amplified, cloned into a PCR-2.1 cloning vector (Invitrogen), and sequenced to confirm the identity of amplicon. The same process was repeated for p74 using p74-specific primers and probes.

Total protein extraction from infected Choristoneura fumiferana larvae Total protein was extracted from fat body of infected larvae at different hours post infection in order to monitor the pattern of ChfuGV ODVP-6E/ODV-E56 and p74 proteins expression. 
Fourth instar Choristoneura fumiferana larvae were infected with 1 $\mu \mathrm{l}$ of purified $\mathrm{ChfuGV}\left(10^{8}\right.$ virus $\left./ \mathrm{ml}\right)$ by force feeding. The infected larvae were then transferred on insect diet and were used for time course expression studies. The fat body of the larvae was harvested at various times post infection and placed in $0.01 M$ Tris, $\mathrm{pH} 7.5$ with $1 \%$ SDS and protease inhibitors $(10 \mu \mathrm{g} / \mathrm{ml}$ each of aprotinin, leupetin, phenylmethylsulfonyl fluoride, $N$-tosyl-phenylalanylchloromethyl ketone, n-tosyl-lysyl chloromethyl ketone; Sigma). The sample was passed through a 27-gauge needle attached to a tuberculin syringe using firm and rapid pressure on the plunger and then was subjected to a freeze-thaw cycle. These two steps were repeated one more time to disrupt the cells.

SDS-PAGE and Western blot analysis Protein samples were disrupted in an equal volume of Laemmli sample buffer (Laemmli, 1970 ) in presence of $\beta$-mercaptoethanol (Bio Rad, Richmond, USA). The samples were then boiled for $5 \mathrm{~min}$ and clarified at $13,000 \mathrm{~g}$ for $5 \mathrm{~min}$ before $10 \%$ sodium dodecyl sulfatepolyacrylamide gel electrophoresis (SDS-PAGE). The protein gels were then electrophoretically transferred to a nitrocellulose membrane (Millipore, Bedford, USA). The membrane was then examined for presence of proteins by staining the membrane with Ponceau S (Sigma, St. Louis, USA). After washing with PBS buffer, membranes were blocked in PBS containing 3\% BSA for 1 $\mathrm{h}$ in room temperature followed by $2 \mathrm{~h}$ incubation with anti-ODVP6E/ODV-E56 (dilution $1: 8000$ ) or anti-p74 (dilution $1: 5000$ ). Membranes were then washed in PBS (pH 7.2), and reacted with the peroxidase-conjugated secondary antibody and visualized using a chemiluminescence substrate (Amersham, Richmond, USA).

\begin{abstract}
Monospecific antisera preparation Gel purified ChfuGV structural proteins were used for production of monospecific polyclonal antibodies. For preparation of antisera purified enveloped nucleocapsids were subjected to electrophoresis on $10 \%$ SDS-PAGE as was explained before. Gels were then lightly (10 min) stained in $0.05 \%$ Coomassie brilliant blue (Sigma) to locate the positions of the protein bands in the gels. After destaining, 39 and $74 \mathrm{kDa}$ bands were excised from the gels and washed in deionized water three times each for $10 \mathrm{~min}$ followed by an over night wash. Following the washes, the preparation of proteins was injected to animals as described by Harlow and Lane (1988). Briefly, the gel pieces were extruded through the barrel of a $5 \mathrm{ml}$ syringe with an equal volume of deionized water to another 5-ml syringe. Antisera were produced in 6 weeks, using male New Zealand rabbits by subcutaneous injection of each antigen at multiple sites along the back. ODVP-6E/ODV-E56 and p74 proteins were injected four times. The initial injection was performed with Freund's complete adjuvant and subsequent injections were done with incomplete Freunds adjuvant.
\end{abstract}

Immunogold localization of ODVP-6E and p74 proteins Immunoelectron microscopy was carried out on complete occluded ChfuGV particles. Purified occluded virions were fixed in $1 \mathrm{ml} 1 \%$ glutaraldehyde/ $1 \%$ paraformaldehyde/phosphate buffer $(0.028 \mathrm{M}$ $\mathrm{Na}_{2}$ HPO4. $2 \mathrm{H}_{2} \mathrm{O}, 0.0387 M \mathrm{KH}_{2} \mathrm{PO}_{4}, \mathrm{pH} 7.2$ ) for $1 \mathrm{~h}$ in room temperature. The sample was then centrifuged in $8,000 \times g$ for 10 min and the pellet was resuspended in $150 \mu \mathrm{l}$ in $0.25 \%$ agarose/ phosphate buffer at $37^{\circ} \mathrm{C}$.
The agarose plug was cut into small blocks and incubated for $1 \mathrm{~h}$ at room temperature. Samples were washed twice in phosphate buffer, each for $10 \mathrm{~min}$. The samples were dehydrated in 10, 30, 50, 70,90 and $100 \%$ ethanol for $15 \mathrm{~min}$ in each concentration. Following dehydration with ethanol, the virus were infiltrated in a $1: 1$ mixture of L. R. White resin and 100\% ethanol for $30 \mathrm{~min}$, and then incubated in three changes of fresh LR White resin for $1 \mathrm{~h}$. The samples then were placed in embedding capsules, which were filled with fresh L. R. White resin and incubated at $52-55^{\circ} \mathrm{C}$ for 12 h. Thin sections were collected on formvar-coated nickel grids.

The grids were incubated in $0.1 \%$ trypsin in PBS buffer for 15 min in order to expose antigenic sites. After washing with distilled water non-specific binding sites were blocked with 3\% BSA in EM-immunogold (EMG) buffer (0.05\% Tween, $0.5 \mathrm{M} \mathrm{NaCl}, 0.01$ $M$ phosphate buffer, $\mathrm{pH}$ 7.2) for $60 \mathrm{~min}$. The sections were then incubated for $90 \mathrm{~min}$ with the primary antibody diluted in EMG with $0.5 \%$ BSA (1: 1000 for both anti ODVP-6E/ODV-E56 and anti-p74). After two rounds of washing with EMG buffer with 0.5 $\%$ BSA, the grids were incubated with goat anti-rabbit antibody conjugated to $10 \mathrm{~nm}$ gold particles (Ted Pella Inc.) for $90 \mathrm{~min}$. Grids were washed twice each in EMG buffer with $0.5 \%$ BSA, PBS, and distilled water and stained for $15 \mathrm{~min}$ with $3 \%$ uranylacetate and $5 \mathrm{~min}$ with lead citrate. TEM was performed using a Philips model 410 transmission electron microscope operating at $80 \mathrm{kV}$.

\section{Results and Discussion}

In a previous study we have reported the identification of genes encoding proteins homologue to ODVP-6E/ODV-E56 and p74 proteins in ChfuGV. In those reports we have analyzed the nucleotide sequences of odvp-6e/odv-e56 and p74 genes and their deduced amino acid sequences (Rashidan et al., 2002; 2003). The genes encoding for these proteins were respectively located in an $11-\mathrm{kb}$ and $8.9-\mathrm{kb}$ BamHI restriction fragments from ChfuGV genomic DNA.

Temporal transcription and translation of ChfuGV odvp6e/odv-e56 gene The open reading frame (ORF) is $1062 \mathrm{nt}$ potentially encoding 353 amino acids, with an estimate molecular mass of $38.5 \mathrm{kDa}$. A late promoter motif (GTAAG) was located at $-14 \mathrm{nt}$ upstream to the first ATG. The presence of a TAAG motif which is a known strong late genes promoter in baculoviruses, signals the strong possibility that $\mathrm{ChfuGV}$ $o d v p-6 e / o d v-e 56$ is expressed during the late phase of the infection cycle.

To examine the temporal nature of odvp-6e/odv-e56 transcription, RT-PCR and Northern blot analyses were performed. RT-PCR analysis on total RNA extracted from infected larvae using the odvp-6e/odv-e56 specific primers detected the presence of a transcript from 24 h p.i. (Fig. 1a). The sequences of the RT-PCR amplicons showed that all amplicons corresponded to ChfuGV odvp-6e/odv-e56 transcripts present in the infected larvae. To substantiate the results obtained by the RT-PCR, Northern blot analysis was performed using a ${ }^{32} \mathrm{P}$-labeled probe. Results of Northern blot 
(A)

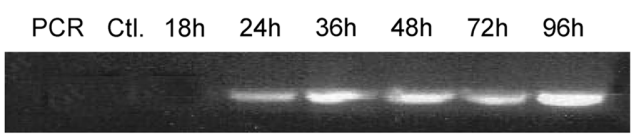

(B)

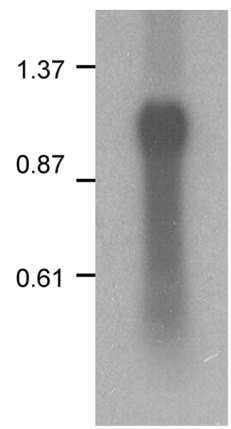

(C)

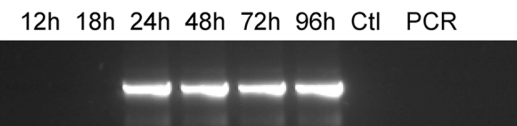

(D)

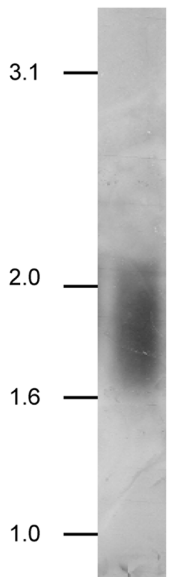

Fig. 1. (A) Analysis of odvp-6e/odv-e56 gene transcripts using RT-PCR using odvp-6e/odv-e56-specific primer sets and total RNA purified from infected Choristoneura fumiferana larvae or uninfected larvae (Ctl.). The result of PCR when the reverse-transcription step is omitted is shown on the first lane. (B) Northern blot analysis on transcripts from infected larvae extracted $48 \mathrm{~h}$ p.i. performed using a ${ }^{32} \mathrm{P}$ labeled DNA probe specific to odvp-6e/odv-e56 revealed the presence of a prominent band detected at $1.2 \mathrm{~kb}$. (C) Analysis of p74 gene transcripts using RT-PCR using p74-specific primer sets and total RNA purified from infected Choristoneura fumiferana larvae or uninfected larvae (Ctl.). The result of PCR when the reverse-transcription step is omitted is shown on the last lane. (D) Northern blot analysis on transcripts from infected larvae extracted $48 \mathrm{~h}$ p.i. performed using a ${ }^{32} \mathrm{P}$ labeled DNA probe specific to p74. Northern blot analysis revealed the presence of a smear extents from 1.7 to almost $2.0 \mathrm{~kb}$.

analysis revealed the presence of a prominent band detected at $1.2 \mathrm{~kb}$ in samples taken from larvae at $48 \mathrm{~h}$ p.i. (Fig. 1b). Studies done on several baculoviral ODVP-6E/ODV-E56 proteins identified them as ODV associated envelope proteins encoded by baculovirus late genes and in all these genes TAAG motifs have been observed in the 5 ' untranslated regions (NTRs) (Braunagel et al., 1996a; Theilman et al., 1996).

In order to characterize the expression pattern of ChfuGV ODVP-6E/ODV-E56 protein, an anti- ODVP-6E/ODV-E56 monospecific antiserum was produced using a SDS-PAGE purified $39 \mathrm{kDa}$ protein. The specificity of this antiserum was examined performing Western blot analysis on ODV enveloped nucleocapsid proteins (data not shown). After confirming its specificity, anti- ODVP-6E/ODV-E56 antisera were used on total protein extracts of infected larvae collected at different times p.i. A band of $39 \mathrm{kDa}$ was first detected at $24 \mathrm{~h}$ p.i. in infected larvae (Fig. 2). Taken together, these data indicate that the ChfuGV ODVP-6E/ODV-E56 is a late protein and is translated from a $1.2 \mathrm{~kb}$ mRNA. The presence of ChfuGV ODVP-6E/ODV-E56 protein at the same time as its transcripts suggests that the expression of this protein is controlled at the transcriptional level.

Temporal transcription and translation of ChfuGV p74 gene The gene has a 1992 nucleotide (nt) open reading frame (ORF) encoding a protein with 663 amino acids with a predicted molecular mass of $74.8 \mathrm{kDa}$. Two late promoter motifs (TAAG and GTAAG) were located upstream of the first ATG of p74 gene. As it mentioned before, the existence of a TAAG motif indicates the elevated likelihood that the gene is expressed during the late phase of the infection cycle.

To examine the temporal nature of p74 transcription, RTPCR and Northern blot analyses were performed. RT-PCR analysis on total RNA extracted from infected larvae using the p74 specific primers detected the presence of a transcript from $24 \mathrm{~h}$ p.i. (Fig.1c). The sequences of RT-PCR amplicons showed that all amplicons corresponded to ChfuGV p74 transcripts present in the infected larvae. To substantiate the results obtained by the RT-PCR, Northern blot analysis was performed using a ${ }^{32} \mathrm{P}$-labeled probe. Results of Northern blot analysis revealed the presence of a band at $2.0 \mathrm{~kb}$ in samples taken from larvae at $48 \mathrm{~h}$ p.i. (Fig. 1d).

In an attempt to examine the expression pattern of $\mathrm{ChfuGV}$ p74 protein an anti-p74 monospecific antisrum was produced using a SDS-PAGE purified $74 \mathrm{kDa}$ protein. The specificity of this antiserum was examined performing Western blot analysis on ODV enveloped nucleocapsid proteins. After confirming its specificity, anti- p74 antisera were used on total protein extracts of infected larvae collected at different times p.i. A band of $74 \mathrm{kDa}$ was first detected at $24 \mathrm{~h}$ p.i. in infected larvae (Fig. 2b). Altogether, these information point to the fact that ChfuGV p74 is a late protein and is translated from a $2.0 \mathrm{~kb}$ mRNA. The presence of ChfuGV p74 protein at the same time as its transcripts, as we have also observed in the 
(A)

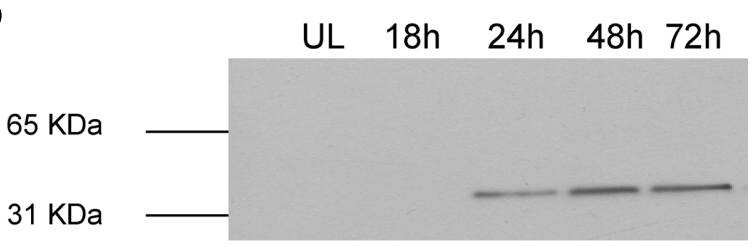

(B)

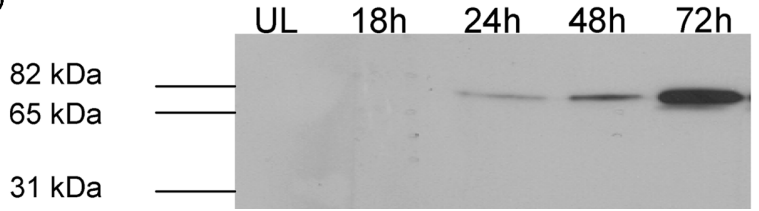

Fig. 2. Preparation of uninfected (UL) or infected larvae with ChfuGV. Immunoblot analysis of ODVP-6E/ODV-E56 and p74 expression in ChfuGV infected fourth instar larvae. Larvae were harvested at different hours post infection (h p.i.). Samples were analyzed by immunoblotting with the anti-ODVP-6E/ODV-E56 antiserum (A). The predominant reaction band was at $39 \mathrm{kDa}$. With the anti-p74 antiserum (B), the predominant reaction band was at $74 \mathrm{kDa}$. Uninfected larvae (UL) were used as control.

case of ChfuGV ODVP-6E/ODV-E56, suggests that the expression of ChfuGV p74 protein is possibly, controlled at the transcriptional level.

\section{Immunolocalization of ChfuGV ODVP-6E/ODV-E56 and} p74 proteins Analysis of ODVP-6E/ODV-E56 hydrophobicity plots suggested the presence of two hydrophobic domains within the C-terminal that were conserved in all baculoviral ODVP-6E/ODV-E56 proteins. These highly hydrophobic regions suggested the existence of two membrane-spanning regions in ChfuGV ODVP-6E/ODV-E56 protein. Same hydrophobic regions were also detected in all other known baculoviral ODVP-6E/ODV-E56 proteins. The locations of these two membrane-spanning regions were almost the same in all baculoviral ODVP-6E/ODV-E56 proteins (Rashidan et al., 2002).

Studies on ChfuGV p74 using various computer assisted prediction tools demonstrated that ChfuGV p74 protein is a membrane protein with two membrane-spanning regions within its C-terminal region. These transmembrane domains were located between amino acid residues 600 to 620 and 633 to 656. In addition to these two regions, an additional transmembrane region was located almost in the center of ChfuGV p74 protein (Rashidan et al., 2003).

To confirm our previous computer-assisted prediction of a membrane location for the ChfuGV ODVP-6E/ODV-E56 and p74 proteins, we performed an immunogold electron microscopy (IEM) analysis on occluded ChfuGV virion using either anti-ODVP-6E/ODV-E56 or anti-p74 monospecific antiserums. ODV was purified and consequently prepared for IEM. The results of IEM confirmed our prediction by localizing the ChfuGV ODVP-6E/ODV-E56 and p74 proteins on the envelope region of the occluded virion (Fig. 3a, b). To
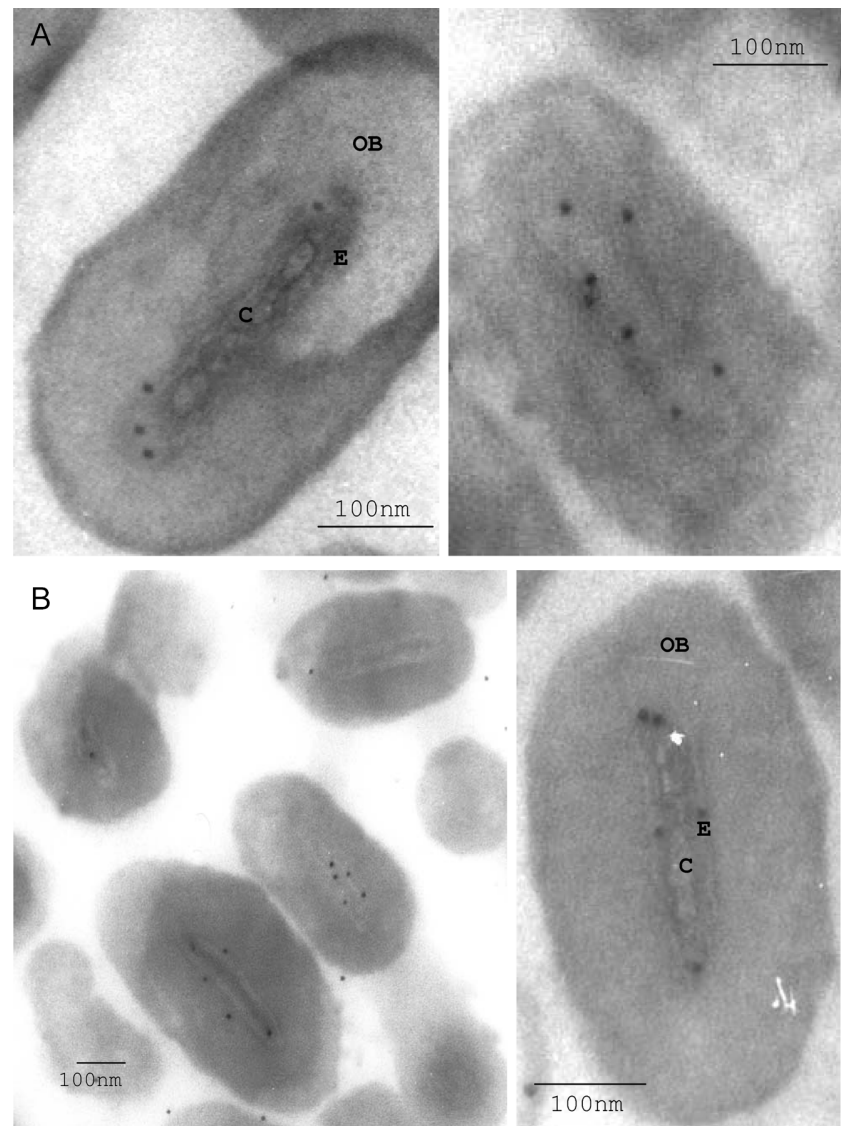

Fig. 3. Immunoelectron microscopy of the ODV phenotype of ChfuGV. Thin sections of occluded virions were incubated with (a) antisera to ODVP-6E/ODV-E56 and (b) antisera to p74 protein, then the grids were incubated with goat anti-rabbit antibody conjugated to $10 \mathrm{~nm}$ gold particles and stained with 3 $\%$ uranyl acetate and $5 \mathrm{~min}$ with lead citrate. The grids then were viewed on a Philips model 410 transmission electron microscope operating at $80 \mathrm{kV}$. Different regions on virion structure is shown OB: occlusion body, E:envelop and C: capsid.

evaluate the accuracy of our IEM method we performed a count of gold beads associated with three regions on individual virions to obtain the total number of beads associated with capsids, envelops and on occlusion bodies of 150 virions. The results in the case of ODVP-6E/ODV-E56 demonstrated that $79 \%$ of beads were associated with envelops while $6 \%$ and $14 \%$ were associated with capsids and occlusion bodies respectively. In the case of this protein the overall average of label density on envelops were roughly 6 and 13 fold greater than the occlusion bodies and the capsids respectively. The results for $\mathrm{p} 74$ revealed almost the same proportions: $86 \%$ of beads were associated with envelops, $6 \%$ associated with capsids and $9 \%$ were localized on occlusion bodies. In the case of $\mathrm{p} 74$ protein the overall average of label density on envelops was roughly 10 and 17 fold greater than the occlusion bodies and the capsids respectively. These findings are in complete agreement with the findings on other baculoviral ODVP-6E/ODV-E56 and p74 homologue proteins. 
Studies carried on AcMNPV, Orgyia pseudotsugata multinucleocapsid nucleopolyhedrovirus (OpMNPV), and Cydia pomonella granulovirus (CpGV) ODVP-6E/ODV-E56 protein homologues all characterized this protein as a late protein associated with the ODV phenotype enveloped nucleocapsid (Braunagel et al., 1996a; Theilmann et al., 1996). The result of immunoelectron microscopy demonstrated that ChfuGV ODVP-6E/ODV-E56 and p74 proteins are indeed bona fide ODV envelope associated proteins.

Similar studies performed on other baculoviruses demonstrated the absence of ODVP-6E/ODV-E56 protein in BV phenotype. Presently, the absence of permissive cell lines for ChfuGV denied us the chance to compare the BV and ODV phenotypes in regard to the ODVP-6E/ODV-E56 and p74 proteins. This would be a very interesting subject to look at in future studies on ODVP-6E/ODV-E56 and p74 proteins. Another attractive study which may shed more light on characterization of ChfuGV ODVP-6E/ODV-E56 and p74 proteins is IEM studies on cells infected with ChfuGV.

Acknowledgments The authors would like to thank Dr. David Morse, Department of Biology, University of Montreal for his scientific discussions. This research was funded by the Ministère des Ressources Naturelles du Québec Grant number 0316-240S to CG.

\section{References}

Adams, J. R. and McClintock J. T. (1991) Baculoviridae, Nuclear polyhedrosis viruses of insects; in Atlas of Invertebrate Viruses, Adams, J. R. and Bonami, J. R. (eds), pp. 87- 204, CRC Press, Boca Raton, USA.

Blissard, G. W. (1996) Baculovirus-insect cell interactions. Cytotechnology 20, 73-93.

Braunagel, S. C. and Summers, M. D. (1994) Autographa californica nuclear polyhedrosis virus, PDV, and ECV viral envelopes and nucleocapsids: structural proteins, antigens, lipid and fatty acid profiles. Virology 202, 315-328.

Braunagel, S. C., Elton, D. M., Ma, H. and Summers, M.D. (1996a) Identification and analysis of an Autographa californica nuclear polyhedrosis virus structural protein of the occlusionderived virus envelope: ODV-E56. Virology 217, 97-110.

Braunagel, S. C., He, H., Ramamurthy, P. and Summers, M. D. (1996b) Transcription, translation, and cellular localization of three Autographa californica nuclear polyhedrosis virus structural proteins: ODV-EI8, ODV-E35, and ODV-EC27. Virology 222, 100-114.

Crook, N. E. (1991) Baculoviridae: subgroup B: comparative aspects of granulosis viruses; in Viruses of Invertebrates, Kurstak, E. (ed.) pp. 73-110, Marcel Dekker, Newyork, USA

Faulkner, P., Kuzio, J., Williams, G. V. and Wilson, J. A. (1997) Analysis of p74, a PDV envelope protein of Autographa californica nucleopolyhedrovirus required for occlusion body infectivity in vivo. J. Gen. Virol. 78, 3091-3100.

Funk, C. J. and Consigli, R. A. (1993) Temporal expression and immunogold localization of Plodia interpunctella granulosis virus structural proteins. Virus Res. 28, 57-66.

Harlow, E. and Lane, D. (1988) Antibodies: A Laboratory Manual, Cold Spring Harbor Press. New York, USA.

Hong, T., Braunagel, S. C. and Summers, M. D. (1994) Transcription, translation, and cellular localization of PDV-E66: a structural protein of the PDV envelope of Autographa californica nuclear polyhedrosis virus. Virology 204, 210-222.

Horton, H. M. and Burand, J. P. (1993) Saturable attachment sites for polyhedron-derived baculovirus on insect cells and evidence for entry via direct membrane fusion. J. Virol. 67, 1860-1868.

Kuzio, J., Jaques, R. and Faulkner, P. (1989) Identification of p74, a gene essential for virulence of baculovirus occlusion bodies. Virology 173, 759-763.

Laemmli, U. K. (1970) Cleavage of structural proteins during the assembly of the head of bacteriophage T4. Nature 227, 680685.

Miller, L. K. (2001) Insect viruses; in Fields Virology, Fields, B. N. et al. (eds.), pp. 533-585, Lippincott-Raven publishers, New York, USA.

Moscardi, F. (1999) Assessment of the application of baculoviruses for control of lepidoptera. Annu. Rev. Entomology 44, 227-289.

Rashidan, K. K., Nassoury, N., Giannopoulos, P. N. and Guertin, C. (2002) Identification and characterization of a conserved baculoviral structural protein ODVP-6E/ODV-E56 from Choristoneura fumiferana granulovirus. J. Bioch. Mol. Biol. 35, 595-603.

Rashidan, K. K., Nassoury, N., Tazi, S., Giannopoulos, P. N. and Guertin, C. (2003) Choristoneura fumiferana Granulovirus p74 protein, a highly conserved baculoviral envelope protein. $J$. Biochem. Mol. 36, 475-487.

Russell, R. L. and Rohrmann, G. F. (1993) A 25-kDa protein is associated with the envelopes of occluded baculovirus virions. Virology 195, 532-540.

Sambrook, J., Fritsch, E. F. and Maniatis, T. (1989) Molecular Cloning: a Laboratory Manual, 2nd ed. Cold Spring Harbor Laboratory Press, New York, USA.

Slack, J.M., Dougherty, E.M., and Lawrence, S.D. (2001) A study of the Autographa californica multiple nucleopolyhedrovirus ODV envelope protein p74 using a GFP tag. J. Gen. Virol. 82, 2279-2287.

Tanada, Y. and Hess, R. T. (1991) Baculoviridae, Granulosis viruses. In Atlas of Invertebrate Viruses, Adams, J. R. and Bonami, J. R. (eds.), pp. 227-257, CRC Press, Boca Raton, USA.

Theilmann, D. A., Chantler, J. K., Stewart, S., Flipsen, H. T. M., Vlak, J. M. and Crook, N. E. (1996) Characterization of a highly conserved baculovirus structural protein that is specific for occlusion-derived virions. Virology 218, 148-158.

Whitford, M. and Faulkner, P. (1993) Nucleotide sequence and transcriptional analysis of a gene encoding gp 41 , a structural glycoprotein of the baculovirus Autographa californica nuclear polyhedrosis virus. J. Virol. 67, 2427.

Wu, W. W., Wang, J. W., Xie, F., Long, Q. X. and Wang, X. Z. (2003) Baculovirus p74 gene is a species-specific gene. Sheng Wu Hua Xue Yu Sheng Wu Wu Li Xue Bao (Shanghai) 35, 834-840. 